\title{
Risk-based maternal group B Streptococcus screening strategy is compatible with the implementation of neonatal early-onset sepsis calculator
}

Niek B. Achten, MD ${ }^{1,2}$ J. Wendelien Dorigo-Zetsma, MD, PhD ${ }^{3}$, Annemarie M.C. van Rossum, MD, PhD ${ }^{4}$, Rianne Oostenbrink MD, PhD ${ }^{5}$, Frans B. Plötz, MD, PhD ${ }^{1,2}$

${ }^{1}$ Department of Paediatrics, Tergooi Hospitals, Blaricum, The Netherlands; ${ }^{2}$ Department of Paediatrics, Amsterdam University Medical Center, Amsterdam, The Netherlands; ${ }^{3}$ Department of Microbiology, Tergooi Hospitals, Blaricum, The Netherlands; ${ }^{4}$ Division of Infectious Diseases and Immunology, Erasmus MC-Sophia Children's Hospital, Rotterdam, The Netherlands; ${ }^{5}$ Department of General Pediatrics, Erasmus MC-Sophia Children's Hospital, Rotterdam, The Netherlands

Background: The early-onset sepsis (EOS) calculator was developed and validated in a setting with routine-based group B Streptococcus (GBS) screening.

Purpose: The study aimed to evaluate the extent of influence exerted by risk-based GBS screening on management recommendations by the EOS calculator.

Methods: All newborns with a gestational age greater than 35 weeks were screened for EOS risk factors in a Dutch regional teaching hospital using a risk-based GBS screening strategy. We calculated the EOS risk at birth and stratified the infants into the following 3 risk levels with corresponding management recommendations: low, <0.65; intermediate, 0.65-1.54; and high, $>1.54$ per 1000 live newborns. Thereafter, we recalculated the EOS risk and recommendation for the newborn infants without available maternal GBS screening results at birth.

Results: In one year, 1,877 eligible births occurred; of them, 206 infants were included. Maternal GBS status was available for 28 of 206 infants (14\%) at birth, while a definitive GBS status was later available for 162 of 206 infants (79\%). Median EOS risk was slightly lower after definitive GBS status was determined ( 0.41 vs. 0.46 per 1,000 live births, $P=0.004$ ). In 199 of 206 newborn infants (97\%), the EOS calculator recommendation remained unchanged after the GBS results unavailable at birth were updated to definitive GBS status. Use of GBS status at birth versus definitive GBS status did not result in the withholding of antibiotic treatment of the newborn infants included in this study.

Conclusion: Risk-based GBS screening is compatible with EOS calculator recommendations. Larger studies are needed to develop the best strategy for combining GBS screening and EOS calculator recommendations.

Key words: Early-onset sepsis, Group B Streptococcus, Maternal screening, Neonatal, Sepsis calculator

\begin{abstract}
Key message
Question: To what extent does risk-based Group B Streptococcus (GBS) screening influence management recommendations by the early-onset sepsis (EOS) calculator?

Finding: In 97\% of the newborn infants, the EOS calculator recommendation remained unchanged after the GBS status at birth was updated to the definitive GBS status.

Meaning: Risk-based GBS screening results are compatible with EOS calculator recommendations.
\end{abstract}

\section{Introduction}

Maternal colonization with Group B Streptococcus (GBS) is a risk factor for neonatal sepsis, as vertical transmission in utero or during labor can result in a life-threatening early-onset sepsis (EOS) in the newborn infant. ${ }^{1)}$ In the United States, GBS colonization is therefore extensively determined among pregnant women, with screening rates at $85 \%$ or higher. ${ }^{2)}$ This universal routine-based screening strategy contrasts with the heterogeneous practice worldwide. For example, in Europe various guidelines advocate risk-based GBS screening depending on obstetric history, whereas in Korea guidelines for the screening of pregnant women for GBS still need to be established. ${ }^{3-7)} \mathrm{A}$ recent study found a GBS colonization rate of $11.6 \%$ among pregnant Korean women and recommended GBS screening and the administration of intrapartum antibiotic prophylaxis (IAP) in pregnant Korean women. ${ }^{4}$ ) The status of maternal vaginal or rectal GBS colonization needs to be available in labor to allow timely administration of IAP to prevent EOS.3) Partly attributed to IAP, a decline in the incidence of EOS to less than 0.5 in 1,000 live births has been observed among term infants. ${ }^{8}$ However, the reported number of infants receiving empiric antibiotic therapy for suspected EOS is much higher. ${ }^{9)}$

To improve the accuracy of empirical antibiotic administration

Corresponding author: Frans B. Plötz, MD, PhD. Department of Pediatrics, Tergooi Hospitals, Rijksstraatweg 1, 1261 AN Blaricum, The Netherlands 凶E-mail: fbplotz@tergooi.nl,fb.plotz@gmail.com, https://orcid.org/0000-0003-3212-2048

Received: 15 January, 2020, Revised: 14 April, 2020, Accepted: 15 April, 2020

This is an open-access article distributed under the terms of the Creative Commons Attribution Non-Commercial License (http://creativecommons.org/licenses/by$\mathrm{nc} / 4.0 /$ ) which permits unrestricted non-commercial use, distribution, and reproduction in any medium, provided the original work is properly cited.

Copyright (c) 2020 by The Korean Pediatric Society 
in newborn infants at risk for EOS, the neonatal EOS calculator was designed and validated in the United States. It has proven to be effective in limiting antibiotic treatment in suspected EOS in term and near-term infants without apparent adverse effects. ${ }^{10-15)}$ The sepsis calculator uses maternal GBS status at birth as 1 of 5 maternal risk factors. The EOS calculator allows 'positive,' 'negative,' and 'unknown' as input for maternal GBS status in the algorithm. In contrast to a routine-based GBS screening, risk-based GBS screening carries the possibility that maternal GBS colonization information is unavailable at time of birth. Often, the GBS culture results become later available after birth. As the EOS calculator is being evaluated for implementation in European and Asian practice, it is important to know whether implementation is compatible with risk-based GBS screening.

The aim of this study was to evaluate to what extent a riskbased GBS screening influences management recommendations by the EOS calculator. If our results indicate that antibiotic recommendations are not influenced by this strategy than the sepsis calculator may also be implemented in countries with a risk-based GBS screening policy.

\section{Methods}

\section{Study population}

We used data from our EOS calculator implementation study in a Dutch regional teaching hospital (Tergooi Hospital). ${ }^{15}$ ) Briefly, we prospectively screened during a single year (April 2016 through March 2017) all newborn infants born at a gestational age of 35 weeks or more, for risk factors or clinical signs of EOS. EOS risk factors included prematurity, maternal fever $\left(\geq 38^{\circ} \mathrm{C}\right)$, positive maternal GBS status, rupture of mem. branes for more than 24 hours before birth, and presumed chorioamnionitis with or without IAP. Newborn infants were included if either one or more maternal risk factors for EOS and/or clinical signs of EOS were present. All data regarding maternal risk factors and the results of the physical examination of the newborn infant were collected through a case report form by the attending physician. Exclusion criteria were birth outside Tergooi Hospital. This study was approved by the Scientific Review Committee of Tergooi Hospitals (study number 15.58; letter reference $\mathrm{kV} / 15.69$ ).

\section{GBS screening protocol}

According to Dutch national guidelines, maternal GBS screening was performed depending on obstetric history (risk-based GBS screening). Screening was performed in case of prematurity, defined as a gestational age less than 37 weeks, rupture of mem. branes for more than 18 hours, maternal fever higher than $38^{\circ} \mathrm{C}$ during delivery, or a history of a previous child with GBS-related disease. ${ }^{16)}$ The Dutch guidelines advise IAP in case of a GBSpositive urine culture to prevent GBS-disease. ${ }^{5)}$

\section{EOS calculator}

The EOS calculator is a multivariate risk assessment based on 5 maternal objective risk factors and the newborn infant's clinical condition to estimate each infant's risk of EOS. The 5 maternal risk factors are gestational age, duration of rupture of membranes, highest intrapartum maternal temperature, use of intrapartum antibiotics (considered adequate prophylaxis if administered more than 4 hours before birth), and maternal GBS status. The EOS calculator allows 'positive,' 'negative,' and 'unknown' as input for maternal GBS status in the algorithm. We used 'unknown' in cases where GBS test results were unavailable at time of EOS calculator application. To assess the clinical condition attending physicians were trained to define the newborn infant's clinical appearance status ('well-appearing,' 'equivocal exam,' or 'clinical illness') according to definitions published along with the EOS calculator. ${ }^{17,18)}$ For each included infant we calculated the EOS risk at birth, using a prior EOS probability of 0.6 per 1,000 livebirths, and we stratified newborn infants into 3 levels of risk (low: $<0.65$; intermediate: $0.65-1.54$; high:> 1.54 per 1,000 live newborns) with a corresponding recommendation on management. This recommendation consisted of standard care, monitoring of vital signs every 3 hours, or the start of empirical antibiotic therapy, respectively.

\section{Data analysis}

For this study, we retrospectively collected the results of all maternal blood, urine, genital and anorectal cultures performed at any time during pregnancy or delivery. We calculated EOS risk and recommendation for each included newborn infant after birth (Fig. 1). We recalculated the EOS risk and recom. mendation for those newborn infants in whose mothers the

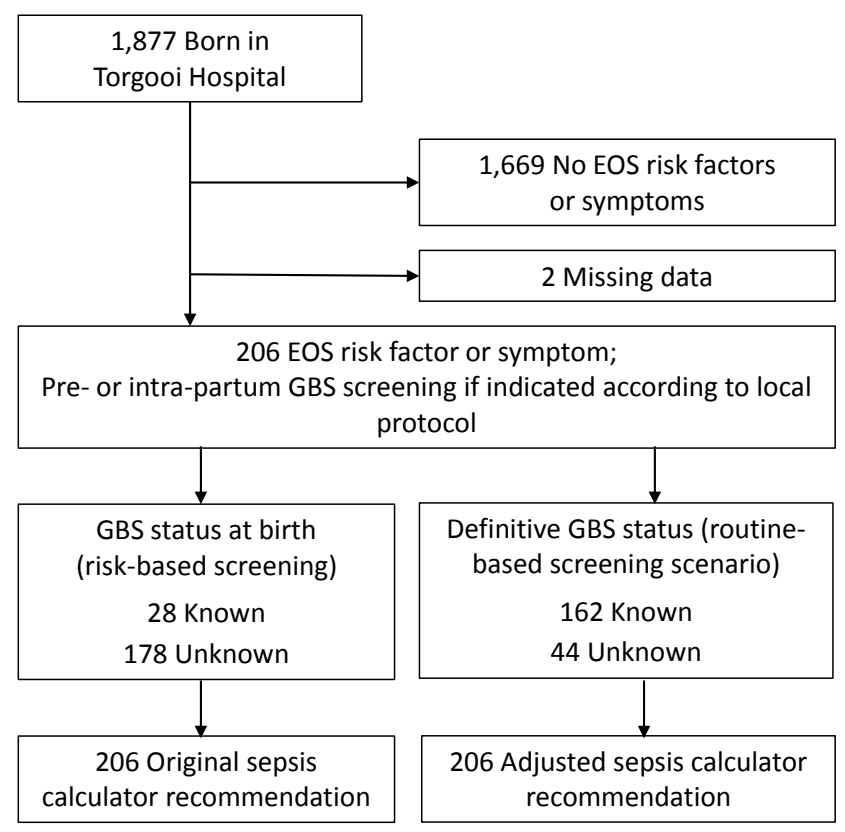

Fig. 1. Study design and inclusion criteria. Flowchart of study design and inclusion criteria. Numbers denote the number of newborns. EOS, earlyonset sepsis; GBS, group B Streptococcus. 
GBS culturing results were unavailable at time of birth. This was defined as definitive GBS status. We compared EOS risk and recommendation at birth and after completing the definitive GBS status. We used Wilcoxon signed-rank test with an alphalevel set at $P<0.05$ to determine statistical significance. Data were analyzed using IBM SPSS Statistics ver. 25.0 (IBM Co., Armonk, NY, USA).

\section{Results}

\section{Patients}

During the study period, 1,877 eligible births occurred. Among these, 208 newborn infants fulfilled the inclusion criteria and 206 had sufficient data to be included in the analysis. Inclusion was due to the presence of one or more maternal risk factors in 183 of 206 (89\%), the presence of clinical symptoms in the newborn infant in 13 of $206(6 \%)$, or a combination of both in 11 of 206 (5\%). Newborn infants were predominantly male (57\%), median gestational age was 39 weeks (interquartile range, $37-40$ weeks).

Maternal GBS status was available for 28 of 206 (14\%) of included newborn infants at birth, mainly from cultures performed during pregnancy. A definitive GBS status was determined for 162 of 206 newborn infants (79\%) (Table 1). Twelve of these included a positive urine culture, of which $6(50 \%)$ were adequately treated with intrapartum antibiotics.

\section{GBS status and EOS calculator results}

We compared EOS risk and recommendation as generated by the EOS calculator at birth and after completing a definitive GBS status (Table 1). Median EOS risk was slightly lower using the definitive GBS status ( 0.41 vs. 0.46 per 1,000 live births, $P=0.004)$. EOS calculator recommendation changed in 7 of 206 (3\%) of newborn infants. Three were assigned a recommendation for clinical observation using vital signs, instead of 'no additional care'; this was the opposite for another 3 infants. Use of the definitive GBS status did not lead to additional re-

\begin{tabular}{|c|c|c|c|}
\hline $\begin{array}{l}\text { GBS status and } \\
\text { EOS calculator results }\end{array}$ & $\begin{array}{l}\text { GBS status } \\
\text { at birth } \\
(n=206)\end{array}$ & $\begin{array}{l}\text { Definitive } \\
\text { GBS status } \\
(n=206)\end{array}$ & $P$ value \\
\hline \multicolumn{4}{|l|}{ GBS status } \\
\hline Positive & 19 & 33 & N/A \\
\hline Negative & 9 & 129 & \\
\hline Unknown & 178 & 44 & \\
\hline EOS risk, median (IQR) & $0.46(0.19-0.89)$ & $0.41(0.17-0.85)$ & 0.004 \\
\hline \multicolumn{4}{|l|}{ Recommendation } \\
\hline NAC & 141 & 142 & 0.655 \\
\hline Vitals every 3 hours & 38 & 37 & \\
\hline Start empiric antibiotics & 27 & 27 & \\
\hline
\end{tabular}

commendations for empirical antibiotic therapy; in 1 case the recommendation changed from antibiotic treatment to clinical observation (Fig. 2).

\section{Discussion}

As use of the EOS calculator spreads to areas without universal screening for GBS, it is important to consider how use of other screening strategies may impact recommendations by the EOS calculator. In particular, it is important to address whether the lack of universal GBS screening may lead to fewer recommendations of antibiotic therapy. We found that in $97 \%$ of newborn infants at risk for EOS in a single Dutch center, the EOS calculator recommendation remained unchanged after we recalculated the EOS risk using the definitive GBS results, which were not yet available at birth. Median EOS risk was slightly lower after recalculation using the definitive GBS screening results. Most importantly, additional knowledge on the GBS status did not increase the total number of recommendations for antibiotic treatment in newborn infants with a GBS-positive mother. In other words, the risk-based screening method did not withhold antibiotic treatment in infants at risk for EOS. These observations together indicate that use of a risk-based screening method only marginally influences EOS calculator management recommendations.

These findings correspond with the modest contribution of the GBS status as a risk factor to the total predictive value in the multivariate EOS calculator model. ${ }^{18)}$ They are further explained by the finding that definitive GBS results were mostly negative,

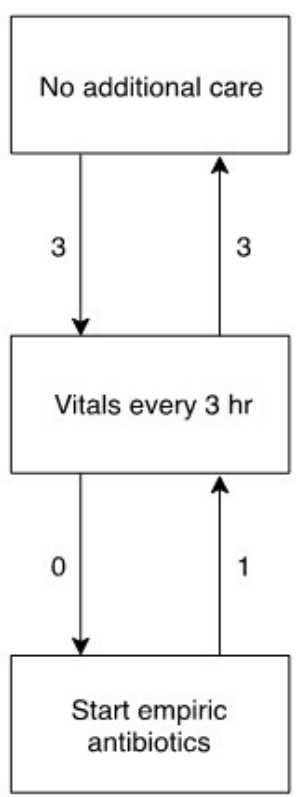

Fig. 2. EOS calculator recommendation changes resulting from definitive GBS status. Changes in EOS calculator recommendation after adjusting for definitive GBS carrier information unavailable at birth using the risk-based GBS screening. Numbers denote the number of newborns affected by the related category change. EOS, early-onset sepsis; GBS, group B Streptococcus. 
thus decreasing EOS risk. In our study population, GBS status was available at birth in only few cases, whereas a definitive GBS status was determined in the vast majority of cases. This indicates that GBS screening shortly prior to birth is common with a risk-based screening strategy, limiting the opportunity to timely administer IAP. A definitive GBS status was not determined in the remaining fifth of our study population, most likely because of absence of indications for testing in obstetric history. Importantly, a substantial part of GBS-related EOS disease occurs despite negative GBS status, ${ }^{19)}$ reflecting that other factors and clinical signs should be taken into account at all times.

Maternal GBS status can mediate the calculated EOS risk and management guided by the EOS calculator indirectly through IAP, which is widely used to decrease GBS-related EOS. ${ }^{20}$ Notable differences across guidelines and in practice exist, ${ }^{5,21)}$ but Dutch guidelines recommend IAP in case of a positive urine culture. ${ }^{5)}$ We found that in our population only $6 \%$ of tested mothers qualified for IAP as a result of a GBS-positive urine culture. Only half of these mothers received adequate intrapartum antibiotics, possibly due to late availability of results of maternal GBS screening.

As use of the EOS calculator spreads to areas with various approaches regarding prevention of GBS neonatal sepsis, this development should be accompanied with thoughtful evaluation of GBS screening strategies. Our findings indicate that use of risk-based GBS screening only marginally impacts EOS calculator antibiotic management recommendation in the new. born infant. However, GBS screening strategies may be most - if not only - helpful when results are provided timely enough for administration of IAP and for use in the EOS calculator. The new generation of rapid intrapartum tests based on polymerasechain-reactions is a promising opportunity in this matter. ${ }^{22,23)}$ Rapid availability of results will be more suitable for guiding decisions on timely IAP and for direct use in the EOS calculator at birth.

Among the strengths of our study is its thorough data collection on maternal GBS status. Also, to our knowledge, this is the first study to evaluate the important difference in GBS screening strategies in the context of the increasingly adopted EOS calculator. Limitations include the single-center study design, which means that results may be different in settings with different risk-based screening strategies. It was conducted in a high-risk population subset with limited sample size, selected on presence of maternal EOS risk factors or neonatal clinical EOS symptoms. The results therefore cannot be generalized to the general newborn infant population without precautions. However, the remaining population of the newborn infants in our hospital had no known EOS risk factors or clinical EOS symptoms. It is therefore at low risk for EOS, and thus unlikely to receive an EOS calculator recommendation for empiric antibiotic therapy, irrespective of maternal GBS status. Hence, we are confident that our design included the vast majority of relevant births where GBS status could have a significant role in decisions regarding empiric antibiotic therapy, making the results indicative for the larger population. Finally, although these results are supportive of the applicability of the EOS calculator in settings with a risk-based screening strategy, this study does not validate the EOS calculator itself for such settings.

In conclusion, our results indicate that use of risk-based GBS screening is compatible with use of the EOS calculator. Larger studies are needed to address the best way of combining GBS screening with use of the EOS calculator.

\section{Conflicts of interest}

No potential conflict of interest relevant to this article was reported.

\section{Acknowledgments}

The authors thank Ellen Tromp (Department of Epidemiology and Statistics, St Antonius Hospital, The Netherlands) for her assistance with statistical analysis.

See the commentary "Utility of neonatal early-onset sepsis calculator in risk-based group B Streptococcus screening approach" via https://doi.org/10.3345/cep.2020.00500.

\section{References}

1. Shane AL, Sánchez PJ, Stoll BJ. Neonatal sepsis. Lancet 2017;390:177080 .

2. Verani JR, McGee L, Schrag SJ; Division of Bacterial Diseases, National Center for Immunization and Respiratory Diseases, Centers for Disease Control and Prevention (CDC). Prevention of perinatal group B streptococcal disease--revised guidelines from CDC, 2010. MMWR Recomm Rep 2010;59:1-36.

3. Di Renzo GC, Melin P, Berardi A, Blennow M, Carbonell-Estrany X, Donzelli GP, et al. Intrapartum GBS screening and antibiotic prophylaxis: a European consensus conference. J Matern Fetal Neonatal Med 2015;28:766-82.

4. Kim DH, Min BJ, Jung EJ, Byun JM, Jeong DH, Lee KB, et al. Prevalence of group $\mathrm{B}$ streptococcus colonization in pregnant women in a tertiary care center in Korea. Obstet Gynecol Sci 2018;61:575-83.

5. NVOG (Nederlandse Vereniging voor Obstetrie en Gynaecologie), NVK (Nederlandse Vereniging Kindergeneeskunde). Preventie en behandeling van early-onset neonatale infecties (Adaptatie van de NICE-richtlijn). Utrecht (the Netherlands): Nederlandse Vereniging voor Obstetrie en Gynaecologie, 2017.

6. Homer CS, Scarf V, Catling C, Davis D. Culture-based versus riskbased screening for the prevention of group B streptococcal disease in newborns: a review of national guidelines. Women Birth 2014;27:46-51.

7. Hong JY, Kim SH, Kim SM, Yee CA, Choi SJ, Oh S, et al. Evaluation of the early onset neonatal sepsis according to two antenatal group B Streptococcus screening methods: risk-based versus universal screening . Perinatology 2019;30:200-7.

8. Mukhopadhyay S, Puopolo KM. Neonatal early-onset sepsis: epidemiology and risk assessment. Neoreviews 2015;16:e221-30.

9. Benitz WE, Wynn JL, Polin RA. Reappraisal of guidelines for management of neonates with suspected early-onset sepsis. J Pediatr 2015;166:1070-4.

10. Kuzniewicz MW, Walsh EM, Li S, Fischer A, Escobar GJ. Development and implementation of an early-onset sepsis calculator to guide antibiotic management in late preterm and term neonates. Jt Comm J Qual Patient Saf 2016;42:232-9.

11. Kuzniewicz MW, Puopolo KM, Fischer A, Walsh EM, Li S, Newman TB, et al. A Quantitative, risk-based approach to the management of neonatal 
early-onset sepsis. JAMA Pediatr 2017;171:365-71.

12. Warren S, Garcia M, Hankins C. Impact of neonatal early-onset sepsis calculator on antibiotic use within two tertiary healthcare centers. J Perinatol 2017;37:394-7.

13. van Herk W, Stocker M, van Rossum AM. Recognising early onset neonatal sepsis: an essential step in appropriate antimicrobial use. J Infect 2016;72 Suppl:S77-82.

14. Kerste M, Corver J, Sonnevelt MC, van Brakel M, van der Linden PD, $\mathrm{M}$ Braams-Lisman BA, et al. Application of sepsis calculator in newborns with suspected infection. J Matern Fetal Neonatal Med 2016;29:3860-5.

15. Achten NB, Dorigo-Zetsma JW, van der Linden PD, van Brakel M, Plötz FB. Sepsis calculator implementation reduces empiric antibiotics for suspected early-onset sepsis. Eur J Pediatr 2018;177:741-6.

16. NVOG (Nederlandse Vereniging voor Obstetrie en Gynaecologie), Nvog. Preventie van neonatale groep-b-streptokokkenziekte (gbs-ziekte). Utrecht (the Netherlands): Nederlandse Vereniging voor Obstetrie en Gynaecologie, 2008.

17. Escobar GJ, Puopolo KM, Wi S, Turk BJ, Kuzniewicz MW, Walsh EM, et al. Stratification of risk of early-onset sepsis in newborns $\geq 34$ weeks' gestation. Pediatrics 2014;133:30-6.

18. Puopolo KM, Draper D, Wi S, Newman TB, Zupancic J, Lieberman E, et al. Estimating the probability of neonatal early-onset infection on the basis of maternal risk factors. Pediatrics 2011;128:e1155-63.

19. Nanduri SA, Petit S, Smelser C, Apostol M, Alden NB, Harrison LH, et al. Epidemiology of invasive early-onset and late-onset group b streptococcal disease in the United States, 2006 to 2015: multistate laboratory and population-based surveillance. JAMA Pediatr 2019;173:224-33.

20. Ohlsson A, Shah VS. Intrapartum antibiotics for known maternal Group B streptococcal colonization. Cochrane Database Syst Rev 2013: CD007467.

21. National Institute for Health and Clinical Excellence (NICE). Neonatal infection (early onset): antibiotics for prevention and treatment [Internet]. London: NICE; 2012 [cited 2018 Jun 19]. Available from: https://www. nice.org.uk/guidance/cg149/resources/neonatal-infection-early-onsetantibiotics-for-prevention-and-treatment-35109579233221.

22. Björklund V, Nieminen T, Ulander VM, Ahola T, Saxén H. Replacing risk-based early-onset-disease prevention with intrapartum group B streptococcus PCR testing. J Matern Fetal Neonatal Med 2017;30:36873.

23. Plainvert C, El Alaoui F, Tazi A, Joubrel C, Anselem O, Ballon M, et al. Intrapartum group $B$ Streptococcus screening in the labor ward by Xpert ${ }^{\circledR}$ GBS real-time PCR. Eur J Clin Microbiol Infect Dis 2018;37:265-270.

How to cite this article: Achten NB, Dorigo-Zetsma JW, van Rossum AMC, Oostenbrink R, Plötz FB. Risk-based maternal group B Streptococcus screening strategy is compatible with the implementation of neonatal early-onset sepsis calculator. Clin Exp Pediatr 2020;63:406-10. https://doi.org/10.3345/cep. 2020.00094 